\title{
中国对虾促雄腺形态结构和功能的初步研究 *
}

\author{
李富花 相建海 \\ (中国科学院海洋研究所, 青岛 266071)
}

\section{关钺词中国对虾 促雄腺 形态结构 功能}

动物的性别控制是生物技术的热点之一, 对虾的性控更是水产养殖高新技术研究中引人 注目的课题. 在中国对虾 (Penaeus chinensis) 中人为地改变其性别比率, 大大提高雌性对虾的 比例，这无疑是提高对虾养殖产量的一条非常有效的途径. 但是到目前为止, 对于中国对虾性 别分化和决定的机理尚不清楚. 近年来我们从促雄腺入手开展了一些这方面的工作.

促雄腺 (Androgenic gland) 最早是由法国生物学家 Charniaux-Cotton 于 1954 年在端足目 中发现的. 由于它在雄性甲壳动物的初级和次级性征的发育和维持上起着重要作用, 因而日 益受到人们的重视. 科学家们已在许多甲壳动物中证实了它的存在, 但是不同种类, 促雄腺的 分布位置不同, 而且外部形态也有很大差异. 在对虾属中促雄腺的研究进行得很少, 仅李霞等 对中国对虾促雄腺的位置和组织学结构进行过报道 ${ }^{[1]}$, 但对于中国对虾促雄腺的外部形态未 进行详细描述, 中国对虾促雄腺功能的研究尚属空白. 本文对中国对虾促雄腺的外部形态进 行了详细观察, 并对其功能进行了初步研究.

\section{1 材料和方法}

\section{1 形态结构观察}

实验材料是 1991 年 7 11 月采集的, 主要取自本所水族楼人工培育的中国对虾(体长 7 $\mathrm{cm}$ 左右), 同时也收集了黄岛养殖场人工养殖的中国对虾(体长 $11 \mathrm{~cm}$ 左右), 以及从胶州湾捕 到的野生雄虾(体长 $14 \mathrm{~cm}$ 左右).

解剖镜观察: 将对虾的精英囊连同相连的一段输精管仔细从体内剥离出来, 在 WILD 解 剖镜下观察其自然色彩和外部形态, 尔后用 Ehrlich 氏苏木精染色, 进行观察和拍照.

扫描电子显微镜观察: 将精英襄及其连接部分先用 $5 \%$ 戊二醛固定 $1 \sim 2 \mathrm{~h}$, 再用酒精、丙 酮等系列脱水, 醋酸异戊酯冲洗, 临界点干燥, 喷金, 然后在 KYKY-1000B 型扫描电子显微镜 下观察、拍照.

组织学观察: 组织学研究的样品用 Bouin 氏液固定, 常规石蜡包埋, 连续切片, 厚度为 7 $\mu \mathrm{m}$, 用 Ehrlich 氏苏木精-伊红染色, OLYMPUS BH-2 显微镜观察并拍照.

\section{2 功能实验}

实验材料为 1993 年在室内人工培育的中国对虾. 共进行了两批手术实验, 第一批手术是

1995-07-20 收稿, 1996-01-04 收修改稿

*国家“㮃登”计划和中国科学院重大攻关项目 
在 6 月 17 日进行的, 手术处理了 76 条虾, 实验虾体长为 $3.4 \mathrm{~cm}$, 当时用肉眼从外观上无法鉴 定对虾的性别. 第二批是在 7 月 16 日进行的, 共处理了 64 条虾, 体长为 $4 \mathrm{~cm}$, 此时有的虾从 外形上隐约可以区分性别, 有的虾尚无法区分. 处理方法是用精细的灼热的铝子将对虾第五 对步足基部形成精英裹的部位破坏, 从而使精英衰及其上的促雄腺组织不能形成. 经手术处 理后的虾放在室内水池继续养至 11 月份, 取出精巢或卵巢, 通过常规的组织学方法观察性腺 发育情况, 另外对手术对虾的外部性征进行仔细观察, 并跟正常虾进行比较.

\section{2 结果}

\section{1 促雄腺的外部形态和内部结构观察}

解剖和比较分析可见中国对虾的促雄腺位于第五对步足基部的肌肉间, 成片状覆盖在精 英衰和射精管连接处, 呈乳白色. 用苏木精染色时, 由于其啫碱性较强, 整个腺体被染成蓝色.

扫描电子显微镜观察可见中国对虾促雄腺是由葡萄形的亚单位组成的, 这些葡萄形亚单 位大小、形态各异(图 1(a)). 亚单位之间以一些形似肌状的结缔组织连接, 使之成为一个统 一的整体. 腺体又通过索状结缔组织与精英衰相连结, 并覆盖在其上(图 1(b)).

组织切片观察表明: 中国对虾促雄腺细胞为圆形或椭圆形,直径约为 $6 \sim 10 \mu \mathrm{m}$, 内有一个 圆形或椭圆形的核, 直径约为 $5 \sim 7 \mu \mathrm{m}$. 细胞最明显的特点是细胞核在细胞中占的相对体积 大, 细胞质较少. 用苏木精-伊红染色, 根据细胞核染色的深浅不同将腺细胞区分为两种类型: 第一类细胞, 数量少, 核的嗜碱性很强, 核质致密, 被苏木精染成深蓝色, 核仁不易分辨; 第二类 细胞, 数量大, 核比较膨大, 啫碱性较弱, 核质比较疏松, 具有明显可见的核仁(图 1(c)). 在不 同大小的虾中, 腺细胞的排列方式有所不同. 在较小的虾中 (体长为 $7 \mathrm{~cm}$ 左右), 细胞主要以 索状排列, 细胞界限较清楚 (图 1(d)). 随着对虾的生长, 细胞由索状排列转变为成团排列; 细 胞团的大小、形态各异, 此细胞团即相当于在扫描电子显微镜下观察到的葡萄形亚单位. 每一 细胞团的外面包以结缔组织的膜, 团内细胞界限不清楚, 细胞核明显可见 (图 1(e)). 细胞团 内有些细胞表现出退化的迹象, 这可能与细胞的分泌活动有关. 在较幼的次成虾中, 促雄腺只 是以细胞索的形式散布在肌肉之间; 随着虾的长大, 腺细胞的数量增加, 腺体逐渐增大, 说明促 雄腺的形成存在一个逐渐发育的过程.

\section{2 功能研究}

第一批经手术处理的虾, 由于手术影响而有部分死亡, 只存活了 50 条, 后来在养殖过程中 又由于自然死亡或生病死亡, 至 11 月份还剩 22 条, 其中雄虾 12 条, 雌虾 10 条, 体长平均为 $9.93 \mathrm{~cm}(8.5 \sim 11 \mathrm{~cm})$. 第二批虾到 11 月份还剩 13 条, 其中雄虾 9 条, 雌虾 4 条, 体长平均为 $9.52 \mathrm{~cm}(8.5 \sim 10.7 \mathrm{~cm})$. 观察比较表明, 经手术处理的这两批虾, 尽管处理开始的时间不同, 但是处理之后这两批虾表现出的特征类似. 组织学切片观察可见, 手术处理过的虾其卵巢或 精巢的组织学结构同对照组比较并无明显的差异, 精巢内有大量精子形成, 卵巢内的卵母细胞 发育正常, 而从外部性征来看却存在有明显的差别. 对照组雄虾 (体长平均为 $9 \mathrm{~cm}$ ), 其交接 器长而宽, 两片连在一起, 交接器长 7 9 $\mathrm{mm}$ (图 1(f)), 而实验组雄虾, 其交接器短而窄, 两片 各自分离, 不能连在一起, 长 $4 \mathrm{~mm}$ 左右 (图 $1(\mathrm{~g})$ ). 除此之外, 第五对步足基部的区域, 对照组 和实验组也有明显差别. 对照组雄虾, 第五对步足基部内侧腹甲上有两个明显的乳突, 而实验 雄虾无. 这说明, 手术处理之后的虾其第二性征的发育明显受到抑制, 特别是交接器显然比对 

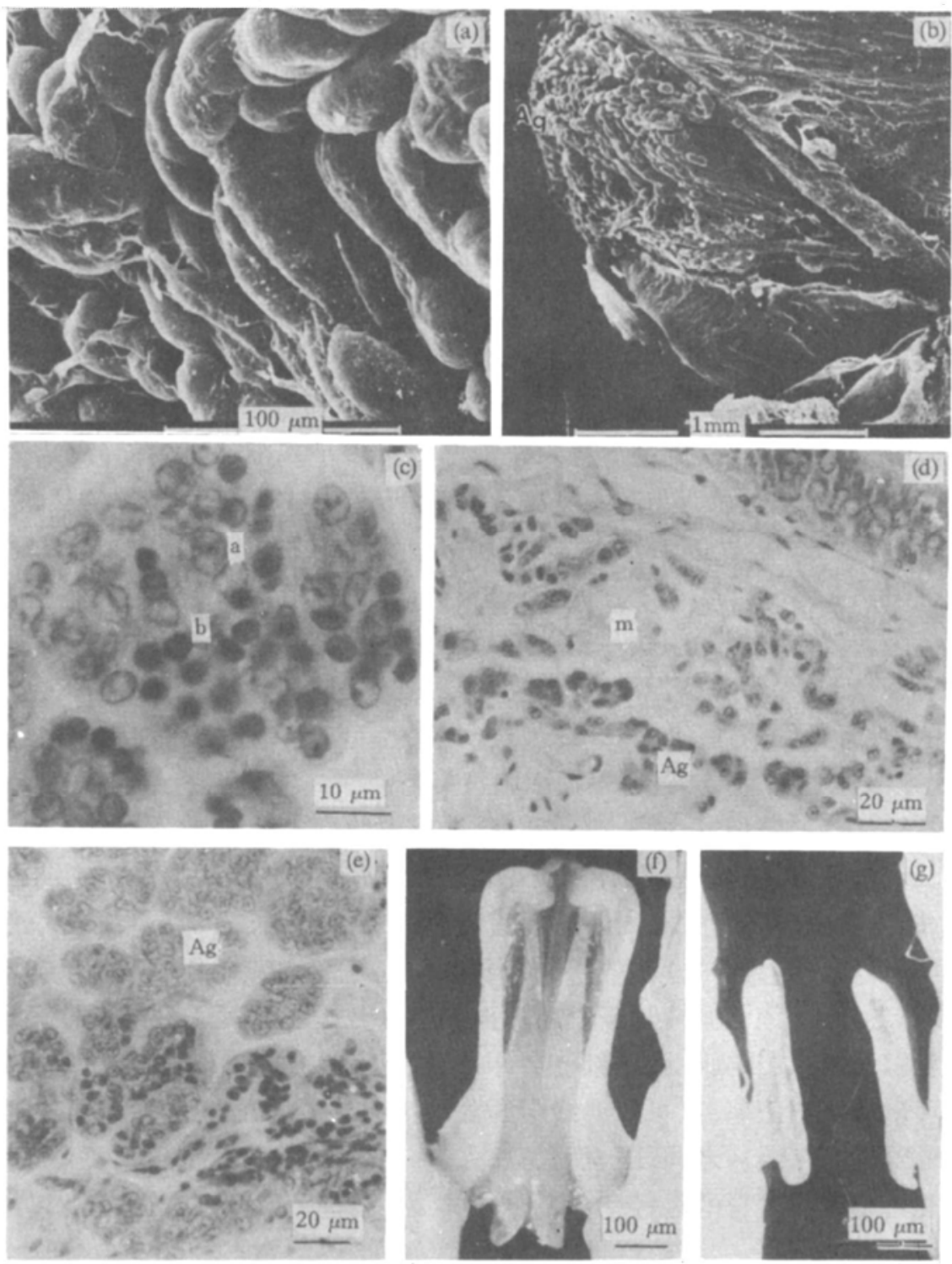

图 1 中国对虾促雄腺形态结构和功能的初步研究

(a)扫描电子显微镜下示促雄腺的蔽萄形亚单位; (b) 扫描电子显微镜下示促雄腺的形态, $\mathrm{Ag}$ 为促雄腺; (c) 组织切 片示促雄腺的两种细胞, 标记为 $a, b$; (d) 组织切片示较小虾中促雄腺腺细胞的排列, $m$ 为肌肉, $\mathrm{Ag}$ 为促雄腺; (e) 组 织切片示较成熟虾中促雄腺腺细胞的排列; ( $\mathrm{f}$ )解剖镜下示对照虾 (体长为 $9 \mathrm{~cm}$ )交接器的形态; (g) 解剖镜下示实 验虾 (体长 $9.5 \mathrm{~cm}$ ) 交接器的形态

照组的小很多.

对实验雌虾而言,纳精襄的形成主要存在两种情况: 第一种情况是纳精襄外形同正常虾; 第二种情况是纳精裳的外形不正常. 这很可能是施行手术对对虾纳精襄形成造成的影响.

\section{3 讨论}

促雄腺在甲壳动物性别发育、性别分化中的重要作用已在许多种类中得到了证实. 在等 
足类 Armadillidium vulgar, 将雄体的促雄腺移植到雌体中引起雌体雄性化; 注射促雄腺的活 性提取物也会引起踓体雄性化 ${ }^{[2]}$. 在罗氏沼虾中也已证明促雄腺的切除会影响个体形态分 化和性别特征 ${ }^{[3,4]}$. 中国对虾促雄腺的重要性是否同已研究的其它种类那样, 显然是一个值 得深入研究的问题, 而详细了解中国对虾促雄腺的位置、形态、结构将是对其功能进行研究的 先决条件.

甲壳动物促雄腺的位置, 尽管都伴生在雄性生殖系统的附近, 但不同种类, 其分布位置不 同 ${ }^{[5]}$ : 有的位于精巢的前端; 有的位于输精管的后端内侧, 有的位于输精管的亚末端; 还有的 位于射精球与输精管的连结处, 附在射精球的内侧. 促雄腺的形状各异, 有的呈缯绕的索状, 有的呈分枝状, 有的呈叶状. 中国对虾促雄腺呈片状覆盖在精荚襄与射精管连接处的内侧面, 与已报道过的其它种类有所不同. 但中国对虾葡萄串状的外形与在克氏原鳌虾 Procambarus clarki 中报道的类似 ${ }^{[6]}$. 不同种类促雄腺的形状不同, 即使同一种类不同的发育时期, 促雄腺 的形态也有很大差异, 这在美洲螯虾 Orconectes nais 促雄腺的形态学和组织学的季节变化的 研究中已有过报道 ${ }^{[7]}$. Joshi 和 Khanna ${ }^{[8]}$ 也报道了淡水溪蟹 Potamon koolooense 的促雄腺的 形态随季节而有所变化. 我们在中国对虾中也观察到了类似的现象. 在较小的虾中, 细胞主 要以索状排列, 细胞界限较清楚, 而在较大的虾中, 细胞主要成团排列, 团内细胞界限不清楚. 从细胞分布状态以及细胞排列形式的不同可以推断在次成虾中, 腺体的分泌活动比较旺盛, 致 使细胞界限不清楚. 从组织学角度看, 中国对虾促雄腺由两种形态的细胞组成, 这与在小龙虾 Procambarus clarki 中研究的情况类似 ${ }^{[6]}$, 而不像美洲螯虾 Orconectes nais 那样可以区分出 5 种类型细胞 ${ }^{[7]}$. 至于这两种细胞各自的功能如何, 尚不清楚, 还需要通过详尽的研究才能推 断.

对促雄腺功能进行的初步实验表明, 促雄腺的破坏对雄虾的交接器的发育会有明显的影 响, 而实验后的雄虾的第五对步足基部形态尽管有所变化, 但因无法排除是否由于手术处理而 造成的, 无法定论. 雌虾中纳精襄的外形不正常可能也与手术的直接破坏作用有关. 这两批 实验虾成熟后生殖腺的组织学结构同对照虾比较无明显不同, 这说明在此两个我们实验的时 刻破坏促雄腺不会影响对虾性腺的发育, 可能与此时对虾的性别已经分化, 生殖腺已经开始形 成有关. 促雄腺的切除对甲壳动物性征的影响在其它种类中也有过报道. Nagamine 等 $^{\left[{ }^{3}\right]}$ 报 道在罗氏沼虾 Macrobrachium rosenbergii 中, 促雄腺的切除会影响它的初级性征和次级性征, 主要依赖于进行手术的时间. 如果进行手术时虾雄性附肢尚未形成, 那么经手术切除促雄腺 后, 雄性附肢将不再形成; 如果进行手术时虾已拥有雄性附肢, 那么经手术切除促雄腺之后, 雄 性附肢将不再失去, 不过其生长率减小. 我们在实验中观察到中国对虾中的情况与罗氏沼虾 中的类似, 把促雄腺破坏后会影响雄性对虾交接器的发育, 使交接器的生长明显受到抑制. 在 手术实验中未观察到促雄腺的切除对初级性征的影响, 可能与我们进行手术的时间较晚有关. 罗氏沼虾的实验表明 ${ }^{[3]}$, 促雄腺的切除会影响初级性征, 如果在最早时期切除促雄腺会出现 完全雌性化, 而在较晚时期切除, 要么部分雌性化, 要么完全无雌性化. 至于在对虾中, 要在很 早时期破坏促雄腺, 手术难度很大, 这主要与对虾本身的生物学特点有关. 中国对虾的促雄腺 在対虾性分化和发育中的作用是否也象在其它某些甲壳动物中所报道的那样, 尚需做进一步 的研究. 
致谢 本工作得到刘瑞玉教授的指导,在此深致谢意.

\section{参考文献}

1 李 霞, 李嘉泳. 中国对虾内分泌器官的一新发现一一促雄性腺。大连水产学院学报, 1993, 8(4):17 21

2 Katakura Y, Hasegawa Y. Masculiniztion of females of the isopod crustacean, Armadillidium vulgare following injections of an active extract of the androgenic gland. General and Comparative Endocrinology, 1983, 48:57 62

3 Nagamine C, Knight A W, Maggenti A et al. Effects of androgenic gland ablation on male primary and secondary sexual characteristics in the Malaysian prawn, Macrobrachium rosenbergii (de Man) (Decopoda, Palaemonidae) with first evidence of induced feminization in a nonhermaphroditic decapod. General and Comparative Endocrinology, 1980, 41:423 441

4 Sagi A, Cohen D, Milner Y. Effect of androgenic gland ablation on morphotypic differentiation and sexual characteristics of male freshwater prawns, Macrobrachium rosenbergii. General and Comparative Endocrinology, 1990, 77:15 22

5 Thampy D M, John P A. The androgenic gland of the shrimp Palaemon dayanus. Marine Biology, 1972, 12:285 288

6 Taketomi Y. Ultrastructure of the androgenic glands of the crayfish, Procambarus clarki. Cell Biology International Reports, $1986,10(2): 131 \sim 137$

7 Carpenter M B, DeRoos R. Seasonal morphology and histochemistry of the androgenic gland of the crayfish, Orconectes nais. General and Comparative Endocrinology, 1970, 15:143 157

8 Joshi P C, Khanna S S. Studies on the androgenic gland of the freshwater crab, Potamon koolooense (Rathbun). Z Mikrosk Anat Forsch Leipzig, 1987, 101(4):699 713 